\title{
Comprensión lectora mediante el uso de la lúdica y la investigación como estrategia pedagógica
}

\section{Reading comprehension through the use of play and research as a pedagogical strategy}

DOI: http://dx.doi.org/10.17981/cultedusoc.9.3.2018.0

\author{
Artículo de investigación. Fecha de recepción: 15/06/2018. Fecha de aceptación: 27/11/2018 \\ Daisy Fernández; Robinson Ruiz; Mónica De La Cruz; Dilia Simanca; \\ Vitelma Pérez; Nevis Cantillo; Yarelis Polo y Albenis De La Hoz ${ }^{1}$ \\ IED Tercera Mixta Sede José Antonio (Colombia)
}

Para citar este artículo:

Fernández, D., Ruiz, R., De La Cruz, M., Simanca, D., Pérez, V., Cantillo, N., Polo, Y. y De La Hoz, A. (2018). Comprensión lectora mediante el uso de la lúdica y la investigación como estrategia pedagógica. Cultura. Educación y Sociedad 9(3), 53-62. DOI: http://dx.doi. org/10.17981/cultedusoc.9.3.2018.0

\section{Resumen}

El impacto que la lúdica tiene sobre la lectura y su comprensión en el proceso de formación, se observa como recurso innovador y por ende diferente como lo es la IEP, que se apropia no solo del proceso sino de las debilidades que se presenta en el proceso de aprendizaje. El estudio tuvo como propósito fortalecer la comprensión lectora mediante el uso de la lúdica y la investigación como estrategia pedagógica. La metodología se orientó bajo el enfoque cualitativo, utilizando el tipo de investigación acción, desde un diseño descriptivo, exploratorio. Para la recolección de datos se utilizaron técnicas como; observación participante y diario de campo. La población estuvo conformada por cuarenta (40) estudiantes de básica primaria de la IED Tercera Mixta sede José Antonio Galán, ubicada en el municipio de Fundación-Magdalena. los resultados arrojaron estrategias basadas en la lúdica y en la investigación para fortalecer la comprensión lectora tales como; lecturas compartidas con amplificación, cronómetro, reyes de la lectura, el plan padrino, ejercicios de comprensión lectora y papá leamos juntos en el aula. Los resultados evidenciaron una marcada deficiencia en los niños y niñas en el área de lengua castellana, más específicamente en el proceso lector y su comprensión.

Palabras clave: Comprensión lectora, lúdica, investigación como estrategia pedagógica.

\section{Abstract}

The impact that playfulness has on reading and its understanding in the training process is seen as an innovative resource and therefore different as is the IEP, which appropriates not only the process but also the weaknesses that arise in the process Learning. The purpose of the study was to strengthen reading comprehension through the use of playfulness and research as a pedagogical strategy. The methodology was oriented under the qualitative approach, using the type of action research, from a descriptive, exploratory design. For the collection of data, techniques such as; participant observation and field diary. The population consisted of forty (40) elementary school students of the Third Mixed IED headquarters, José Antonio Galan, located in the municipality of FundaciónMagdalena. the results yielded strategies based on play and research to strengthen reading comprehension such as; shared readings with amplification, stopwatch, kings of reading, the godfather plan, reading comprehension exercises and dad read together in the classroom. The results showed a marked deficiency in the children in the Spanish language area, more specifically in the reading process and its comprehension.

Keywords: Reading, playful understanding, research as a pedagogical strategy. 


\section{Introducción}

Dentro de las dificultades que inciden en el bajo rendimiento de los estudiantes de la IED Tercera Mixta Sede José Antonio encontramos la comprensión e interpretación de texto como uno de los factores determinantes, y se refleja en la pobre expresión oral y escrita de los estudiantes lo que dificulta las diferentes áreas del conocimiento, viéndose afectado el desarrollo social y emocional de los educandos.

Leer no solo consiste en reconocer y unir letras, el propósito de la lectura es la comprensión de lo que el autor expresa, para lo que se necesita entender e interpretar correctamente; los niños y niñas merece especial atención en relación al tipo de lectura con la que iniciaran este proceso las que deben estar constituidas por lecturas de cuentos infantiles puesto que a través de estas se estimula la imaginación, el lenguaje, la expresión entre otras. Los maestros están en la obligación de buscar y emplear metodologías que lleven a promover hábitos lectores debido a que resulta ser un medio indispensable para la adquisición de la cultura y un puente indispensable para otros aprendizajes.

La lúdica como estrategia para incentivar la investigación en la comprensión lectora de los estudiantes surge en efecto como una necesidad sentida frente a la violencia en las relaciones interpersonales, falta de tolerancia y respeto, agresiones físicas y verbales, matoneo entre niños y niñas de la instituciones educativas, es por esta razón que, la cultura de violencia y la cultura de la paz, es por esta razón una forma de contribuir mediante una construcción de bases sólidas en medio del respeto, la responsabilidad de los docentes, el compañerismo entre los estudiantes, implementando estrategias socializadoras e intermediarios entre la cultura de violencia y la cultura de la paz, con miras pertinentes en la comprensión lectora de los estudiantes

En otras palabras, la lúdicas es un adjetivo que califica todo lo que se relaciona con el juego, derivado en su etimología del latín "ludus" cuyo significado es precisamente juego, como actividad placentera donde el ser humano se libera de tensiones y de las reglas impuestas por la cultura". Siendo "la parte lúdica de los estudiantes el juego, la parte innata del ser humano que aflora durante las actividades que realizan, puede ser experiencias vividas, observadas, potenciando de manera eficiente hasta lograr superar el interés por ejecutar cualquier actividad y dentro del campo educativo la parte lúdica de los educandos juega un papel importante a la hora de consolidar los conocimientos" (Núñez, 2004).

Reveco (2005) señala que "la lúdica se identifica con el ludo que significa acción que produce diversión, placer, alegría y toda acción que se identifique con la recreación y con una serie de expresiones culturales como el teatro, la danza, la música, competencias deportivas, juegos infantiles, juegos de azar, fiestas populares, actividades lúdicas como: la pintura, la narrativa, la poesía entre otros".

En ese sentido, la lúdica no solo fomenta el desarrollo Psico-social, la adquisición de saberes, la conformación de la personalidad, encerrando una amplia gama de acciones donde interactúan el placer, el gozo, la creatividad y el discernimiento. Cabe decir que, la atmósfera que envuelve el ambiente del aprendizaje que se genera específicamente entre maestros y alumnos, docentes y discentes, entre facilitadores y participantes, de esta manera es que en estos espacios se presentan diversas situaciones de manera espontánea, las cuales generan gran satisfacción. La Capacidad lúdica se desarrolla articulando estructuras psicológicas globales tales como las cognitivas, afectivas y emocionales. 
González (2003) afirma que "una estrategia lúdica ayuda a fijar el conocimiento de manera eficiente, fomentando las participaciones activas de los estudiantes durante el proceso enseñanza- aprendizaje, aportando a la asimilación de los saberes obtenidos mediante la producción de ideas, pensando, analizando, reflexionado y sobre todo mediante el desarrollo y la construcción de los saberes mediante la lectura" (p. 56).

En este sentido, para Delgadillo y Chacón (2014) el aprendizaje que se origina de una manera lúdica permite que los niños y las niñas acumulen conocimientos específicos con relación al área de lectura y escritura pues de esta manera le está permitiendo comprender su realidad y seguir aprendiendo según sus propias necesidades.

En efecto, la lectura no solo consiste en reconocer y unir letras entre sí, sino que el propósito de leer es la comprensión de lo que el autor expresa en los textos que escribe, para lo que se necesita entender e interpretar correctamente lo que transmiten; los niños y niñas merece especial atención en relación al tipo de lectura con la que iniciaran este proceso las que deben estar constituidas por lecturas de cuentos infantiles puesto que a través de estas se estimula la imaginación, el lenguaje, la expresión entre otras. La noción de que para la comprensión del texto se debe poder identificar sus componentes específicos de dominio ya fue planteada en 1944 en un trabajo de Davis citado Abusamra, Ferreres, Raiter, de Beni. y Cornoldi (2010).

Estos criterios que forman parte de los procesos cognitivos (percepción, atención, comprensión, memoria) al ser implicados en la adquisición de la lectura y su comprensión, afectan a un grupo de estudiantes del grado 3 de básica primaria que oscilan entre los 5 y 12 años de edad de las IED. Estas dificultades constituyen una gran preocupación para muchos docentes y padres de familia, ya que afectan el rendimiento escolar y las relaciones interpersonales de los alumnos. (Antecedentes de la dificultad, a nivel mundial hasta llegar a nuestro país). Los maestros están en la obligación de buscar y emplear metodologías que lleven a promover hábitos lectores debido a que resulta ser un medio indispensable para la adquisición de la cultura y un puente indispensable para otros aprendizajes.

Los hábitos en la educación son de gran importancia para ayudar a fortalecer los conceptos y así tener éxito en la parte académica, esto se puede evidenciar en los resultados que tienen los estudiantes al dedicar tiempo, atención y esmero a sus labores y actividades escolares. Esto lo respaldamos con la siguiente cita de Quintero (2010). Los hábitos son los que dan estructura y forma a la vida cotidiana del niño, y por supuesto también del adolecente, del joven y del adulto, lo cual nos proporciona a todos seguridad y estabilidad. Sin hábitos adecuados, la vida de cualquier persona se vuelve desorganizada y puede incluso llegar a ser caótica (Quintero, 2007, p.18).

La educación en Colombia se orienta a fortalecer aspectos pedagógicos con diferentes planes y proyectos; aun cuando buscan un solo objetivo "mejorar", llevar la calidad de la educación colombiana a la excelencia han presentado ciertas incongruencias. Estos modelos impuestos por las administraciones de turno tienden a ser estandarizados, lo que conlleva a no ser del todo ajustados y reales en algunos sectores de la educación debido a que cada zona de Colombia tiene diferentes particularidades.

Dentro de los proyectos educativos deben estar inmersas las estrategias metodológicas de enseñanza del educando, herramientas que cada educador debe tener bien claras en el momento de utilizarlas. 
Hoy el Ministerio de Educación Nacional - MEN (República de Colombia. MEN, 2016; 2016b) habla de estándares, lineamientos y competencias, Derechos Básicos de Aprendizaje (DBA), acompañamiento a través del Programa Todos a Aprender (PTA) entre otros, causando cierto grado de confusión en los docentes sobre cómo utilizar estos elementos; más que todo por la fundamentación estandarizada de estas herramientas que en muchos de los casos no se adaptan al medio en que se están aplicando, limitando así su desarrollo asertivo en la enseñanza aprendizaje de los estudiantes.

Es de anotar que se observa como de acuerdo a los resultados arrojados por las pruebas saber 3, 5 aplicadas por el Instituto Colombiano para el Fomento de la Educación Superior - ICFES (2016). Los procesos educativos en el área de lengua castellana; más exactamente en comprensión lectora no son los esperados en la educación del Magdalena, puntualmente en el municipio de Fundación; Análisis Institucional y Pruebas saber (ICFES, 2016), los procesos se ven muy lentos en su asimilación y en algunos casos parece que incluso no se diera dicho aprendizaje.

Por tanto, condiciones internas y externas a los procesos de enseñanza aprendizaje conllevan a enfrentar falencias en la aprehensión de los conocimientos en la comunidad estudiantil, asociada en mayor preponderancia a deficiencias en los procesos lectores y por ende su comprensión. Para esta situación tan compleja y generalizada se considera necesario que los docentes tengan de la mano estrategias pedagógicas que permitan fortalecer la forma como se imparten dichos conocimientos y como el estudiante se adueña o se empodera de él.

En el desempeño como docentes, de acuerdo con las observaciones y seguimientos realizados a un grupo de estudiantes, se notó con preocupación las di- ferentes dificultades que han presentado los estudiantes en lectura y comprensión lectora; convirtiéndose en una problemática académica que va en aumento en las instituciones educativas. Dentro de las dificultades encontradas las más sobresalientes son: desordenes en los procesos cognitivos (percepción, atención y memoria), los cuales se caracterizan por falta de interés y motivación en los alumnos, confusión de letras y fonemas, tienden a olvidar lo aprendido, no trabajar ni participar de algunas actividades lectoras; dicha situación afecta el proceso de enseñanza aprendizaje en el área de lenguaje y sus respectivas asignaturas, que son fundamentales en el desarrollo de otras áreas académicas.

Cuando se trata de buscar explicación a dichos inconvenientes se acude a diferentes herramientas y estrategias para poder ayudar a los alumnos a mejorar las dificultades que manifiestan día a día y así mejorar su aprendizaje lector. Los nuevos tiempos requieren nuevas estrategias y la I E P apoyada en la lúdica resulta imprescindible en la práctica educativa; permitiendo optimizar el aprendizaje, volviéndolo una actividad placentera, facultando al alumno a ser un protagonista activo de dicho aprendizaje y orientando a solucionar las dificultades mencionadas anteriormente.

Se observa que dentro del proceso lector en el aula de clase y en situaciones de comunicación oral y lectura compartida se propone que las relaciones que surgen entre los niños y adultos en situaciones de búsqueda de diálogos en la vida cotidiana son de gran poder de enriquecimiento en el proceso de lectura ya sea de tipo científico, literario entre otras. Nuevamente se resalta la importancia del entorno familiar en el proceso de adquisición del aprendizaje lector puesto que este determinara una gran fuente de preconceptos para el aprendizaje como tal. 
Por otro lado, la postura del constructivismo en el aprendizaje de la lectura. Dentro de estos preconceptos lectores que el niño trae consigo al aula de clases es de relevancia apoyarse en el constructivismo (Vygotsky, 1979), se considera como el marco teórico que ubicó la investigación, observando la orientación que apoyó al análisis, explicación e interpretación del proceso de enseñanza y aprendizaje de la lectura que se desarrolla en la escuela. Por otro lado, asumir el paradigma constructivista implica cambiar la conceptualización de lectura y los métodos de enseñanza-aprendizaje, comprender el sujeto que aprende (estudiantes-lectores), redefinir el papel del docente, mejorar el entorno alfabetizador e innovar en evaluación. De manera tal que la didáctica de la lectura constructivista establece una práctica docente distinta a la tradicional.

Ahora bien, para Vygotsky (1977) el aprendizaje de la lectura considera que para que haya una trasformación en los procesos educativos y por ende un aprendizaje significativo se hace necesario un cambio de fondo en el entorno educativo, en donde el entorno lector sea ubicado como un espacio atractivo para que su función en el aprendizaje lector sea fundamental y de resultados, además de involucrar a la familia en este procesos ya que con ello el entorno lector familiar también será enriquecedor al proceso. Cabe mencionar que las aportaciones de Vygotsky (1977) son de gran relevancia en la actualidad por lo que se convierten en una fuente confiable de consulta más aun en lo referente al aprendizaje de la lectura y su transversalidad en el aula.

Ahora bien, dentro de los procesos actuales de formación en educación es de gran relevancia la planeación adecuada y organizada de dichos procesos, se observa como cada día la preocupación por parte de los docentes en la estructuración de sus temáticas a desarrollar en el queha- cer pedagógico diario es mayor; cada día se improvisa menos y se tecnifica más el desempeño docente. Ahora bien, dentro del desarrollo de los procesos educativos la planeación es un pilar fundamental dentro del quehacer docente diario, debido a que es en ella donde se fundamenta la acción pedagógica en los diferentes contextos además de servir como bitácora al docente en su desempeño.

Por consiguiente, se hace necesario crear una estrategia que fortalezca la cultura del buen trato entre estudiantes, especialmente en las instituciones educativas del departamento del Magdalena, lo que quiere decir que, en esta etapa los niños se agreden por situaciones sencillas de manera verbal y físicamente, en vez de recurrir al diálogo o la conciliación. Esta situación se refleja en los observadores de estudiantes que contienen en su mayoría escritos, compromisos, llamados de atención y descripciones de situaciones de conflicto en clase y en las horas de recreo.

Ante esta situación, se requiere de una mediación pronta y eficaz que destaque la importancia de educarse a convivir, respetando el derecho a la diferencia y aprehendiendo herramientas para la resolución pacífica de los conflictos. A pesar de la problemática descrita, es notable el cambio de actitud de los estudiantes cuando juegan o comparten en equipo, ya sea en clase o en horas de descanso. En otras palabras, esta situación condujo a plantear el problema de investigación desde la pregunta: ¿qué cambios genera la lúdica para incentivar la investigación en la comprensión lectora en los estudiantes? partir del problema planteado, además para brindar a la comunidad educativa un enriquecimiento en el desarrollo de los niños y niñas, brindándoles mejores posibilidades de expresión y satisfacción en las que se entrelazan el goce, la actividad creativa y el conocimiento. 


\section{Metodología}

Se realizó un estudio de tipo cualitativo, utilizando el modelo de investigación acción, desde un diseño descriptivo, exploratorio. La investigación cualitativa busca conocer e interpretar la realidad de los participantes a través de sus propias experiencias, entregando una información subjetiva del fenómeno de estudio (Hernández, Fernández, y Baptista, 2010). Por su parte el modelo de investigación acción plantea una producción de conocimiento basado en la reflexión de los participantes, teniendo en cuenta la participación activa de maestros en el proceso de enseñanza y acompañamiento tecnológico, guiando al estudiante en su proceso de investigación. Generando nuevo saber y conocimiento sobre una realidad determinada en un proceso de aprendizaje colaborativo.

Los estudios con un diseño descriptivo están encaminados a especificar las características de la población sujeto de estudio o los fenómenos que sean sometidos a análisis. Bajo un estudio de tipo exploratorio, debido a que no se han realizado estudios previos en la institución. (Hernández, Fernández, y Baptista, 2010). La población sujeto de estudio estuvo constituida por cincuenta (50) estudiantes, de los grados séptimo y octavo de básica secundaria, con edades comprendidas entre los once (11) y quince (15) años, para seleccionar la muestra se realizó un muestreo no probabilístico de tipo intencional seleccionando directa e intencionadamente los individuos de la población. Tomando como criterios de inclusión estudiantes del grupo de investigación ambiental.

Para la recogida de datos se utilizó la observación participante y el diario de campo, estas son técnicas de recolección cualitativas que implican que el docente tome un rol activo en el proceso de investigación, donde no solo debe estar como espectador del proceso de implementación, sino que también debe participar del mismo, registrando cada elemento observado con relación a la variable de estudio. En el diario de campo se registran y sistematizan los elementos obtenidos de la observación participante donde el contacto con las personas del contexto, modulan las reflexiones y conclusiones que se pueden extraer.

La metodología del proyecto de investigación se realizó a partir de los recorridos de las trayectorias de indagación, basados en la integración de la IEP al aula, articulado al plan de estudios, transversalizando así la educación ambiental a las diversas asignaturas, lo anterior con la finalidad de generar una trasformación social y educativa a través del aprendizaje en contexto, involucrando intereses, motivaciones y realidades de los estudiantes para generar conocimiento científico.

Para identificar el contexto lector de los niños se aplicaron técnicas pedagógicas como la lectura con cronómetro, textos dados para comprensión lectora, luego de la aplicación de estrategias se aplicó medición de la lectura por medio de la observación directa con registro en observadores del grupo focal y sus progresos, además de la coherencia en las reflexiones de lecturas realizadas y de la coherencia en los mapas conceptuales, historietas, producción de párrafos entre otros.

Para la consecución de estos propósitos se estarán desarrollando actividades que por medio de la IEP ayuden a establecer estrategias que ayuden a fomenta la habilidad lectora y la comprensión de la misma en los estudiantes, facilitando un mejor desarrollo en las competencias de aprendizaje.

Dentro de las estrategias a desarrollar tenemos:

a. Lectura compartida, la cual consiste en ubicar dos niños a leer y comparten las reflexiones de dicha lectura. 
b. Lectura con amplificación, en la cual se busca fortalecer la lectura oral, pronunciación y puntuación.

c. Lectura con cronometro, con la cual se busca fortalecer la rapidez y fluidez lectora en los niños.

d. Los reyes de la lectura, con estad actividades se permite que el niño escoja su lectura de acuerdo a su preferencia, con lo cual se elabora un cuadro de honor con los niños que sobresalgan en rapidez, pronunciación, entonación, comprensión y seguridad en el momento de leer.

e. El plan padrino, con el cual los estudiantes que sobresalen en la lectura acogen a los niños que muestren dificultad, con el cual escogen un tema que les interese para realizar lectura de iniciación y luego de profundización con diferentes textos que a su vez transversalizan en otras áreas, incentivándolos a la investigación en todas las áreas desde la práctica de la lectura.

f. Ejercicios de comprensión lectora, con la cual se realiza una lectura en diferentes temas con lo cual el niño explora su autoconocimiento produciendo mapas conceptuales, párrafos, historietas entre otros.

g. Papá leamos juntos en el aula. Por iniciativa de los niños se invita a los padres de estos a un acompañamiento de lectura en el aula de clase, donde los padres no solo hacen parte del proceso sino, que también expresan juicios valorativos acerca de los progresos de sus hijos con respecto a los temas tratados.

\section{Resultados}

Más allá de la complejidad del proceso de comprensión de textos y de las posibles formas de aproximación a las dificultades, hay coincidencia en que el trastorno de la comprensión textual, con diferentes grados de severidad, es un hecho frecuente en la población estudiantil del Magdalena (Abusamra, Ferreres, Raiter, de Beni y Cornoldi, 2010). En los procesos reflexivos de la problemática en la lectura y su comprensión; se toma como base las estrategias pedagógicas de Investigación con las cuales se busca no solo formar a los estudiantes acerca de la mejor forma de solucionar la situación problema, que para este proceso es la deficiencia en lectura y su comprensión; sino también en que los niños tomen la posición de proponentes en las soluciones.

Dentro del proceso de la ejecución de las estrategias de comprensión de lectura se observó cómo los niños se motivaron con las herramientas tecnológicas mientras que los métodos tradicionales no generaron tanto resultado; razón por la cual se establece la utilización de herramientas tecnológicas como una de las estrategias lúdicas para superar las dificultades en la lectura y su comprensión, así como en los procesos de la ejecución del proyecto los padres se van involucrando lo cual hace que el resultado y apropiación del proceso lector sea más notorio; mientras que los chicos que los padres aún no se involucran los procesos son un poco más lento que los que cuentan con el respaldo en casa.

Se observa que en el proceso de ejecución de la presente investigación de forma lenta y gradual los niños se fueron involucrando en el proceso de apropiación de la IEP y su aplicación, con lo cual se logró superar en un gran porcentaje la deficiencia lectora, es decir, se consiguió el logro del objetivo del proyecto. Ahora bien, en este proceso se observó que se necesita más disponibilidad de tiempo para la implementación del proceso, puesto que por las exigencias de los directivos los espacios de aplicación son pocos. A continuación, se sistematizan las actividades realizadas en la ejecución del protecto (tabla1) 
TABLA 1

Sistematización de actividades realizadas en el estudio

Actividad

Descripción

Lectura compartida

Lectura con ampliación

Lectura con cronometro

Los reyes de la lectura

El plan padrino

Ejercicios de comprensión lectora

Papá leamos juntos en el aula
La cual consiste en ubicar a los niños a leer y comparten las reflexiones de dicha lectura.

Se buscó fortalecer la lectura oral, pronunciación y puntuación. Se buscó fortalecer la rapidez y fluidez lectora en los niños. Con esta actividad se permitió que el niño escogiera su lectura de acuerdo a su preferencia, con lo cual se elabora un cuadro de honor con los niños que sobresalgan en rapidez, pronunciación, entonación, comprensión y seguridad en el momento de leer.

Con el cual los estudiantes que sobresalen en la lectura acogen a los niños que muestren dificultad, con el cual escogen un tema que les interese para realizar lectura de iniciación y luego de profundización con diferentes textos que a su vez transversalizan en otras áreas, incentivándolos a la investigación en todas las áreas desde la práctica de la lectura.

Con la cual se realizó una lectura en diferentes temas con lo cual el niño explora su autoconocimiento produciendo mapas conceptuales, párrafos, historietas entre otros.

Por iniciativa de los niños se invita a los padres de estos a un acompañamiento de lectura en el aula de clase, donde los padres no solo hacen parte del proceso sino, que también

Fuente: elaboración propia.

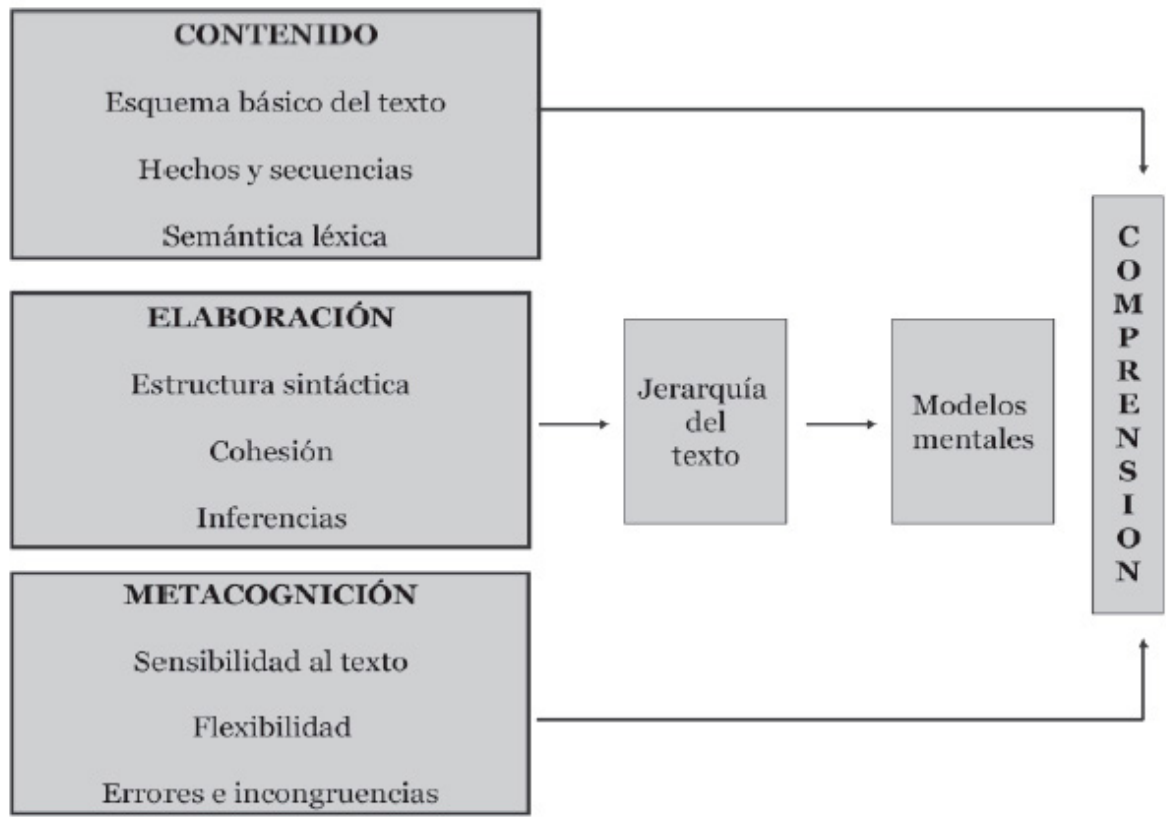

Figura 1. Esquema descriptivo de la relación entre las áreas implicadas en la comprensión de textos.

Fuente: Davis citado por Abusamra, Ferreres, Raiter, de Beni, y Cornoldi, 2010. 
Resulta oportuno decir que, la noción para la comprensión del texto se debe poder identificar sus componentes específicos de dominio ya que planteada en 1944 en un trabajo de Davis citado por (Abusamra, Ferreres, Raiter, de Beni, y Cornoldi, 2010). (figura 1)

\section{Conclusiones}

Con el análisis implementado a estudiantes de las diferentes áreas se demuestra que los mismos tienen problemas en los niveles de lectura ya que manifiestan tener dificultad para comprender lo que leen, además porque los docentes no prestan importancia a la implementación de nuevas estrategias.

Con la implementación de las lecturas los estudiantes de la IED, se verifica que no se está utilizando estrategias lúdicas para mejorar la comprensión lectora, que todavía se sigue utilizando las formas tradicionales de enseñanza, sin una metodología que pueda lograr fijar los aprendizajes de manera eficiente. Se concluye por lo tanto la necesidad de aplicar el uso sistemático y progresivo de estrategias lúdicas para mejorar la comprensión en las lecturas de los estudiantes de la IED, teniendo en cuenta que esta competencia es uno de los pilares fundamentales dentro del proceso de enseñanza y aprendizaje. Se hace evidente que los estudiantes han mejorado en su nivel de lectura comprensiva con la aplicación de las nuevas estrategias lúdicas utilizando la IEP ya que demuestran que los educandos son más participativos y sobre todo analizan, razonan e interpretan los textos que ahora leen.

Por otro lado, se pueden emplear como parte de las IEP a los ambientes virtuales de aprendizaje como entornos educativos mediados por las TICs, en una construcción de saber y conocimiento donde se encuentra la disposición de los materiales y la organización, como también la distribución de los recursos didácticos, el manejo del tiempo y las interacciones que se dan en el aula. En ese sentido los ambientes virtuales de aprendizaje son espacios de socialización e interacción entre docente y estudiante, donde pueden poner en común el desarrollo de distintas actividades para su seguimiento y evaluación. Esto facilitando la comunicación, el procesamiento y distribución de información y creando nuevas posibilidades y limitaciones para el aprendizaje de la comprensión lectora.

\section{Referencias}

Abusamra, V., Ferreres, A., Raiter, A., De Beni, R. y Cornoldi, C. (2010). Test Leer para Comprender. Buenos Aires: Paidos.

Delgadillo, A. y Chacón, J. (2014). La lúdica como estrategia didáctica para el fortalecimiento de la lectura y la escritura. [Tesis]. Coorporación Universitaria Minuto de Dios. Bogotá, D.C.

González, B. (2003). Fundamentación pedagogica del curriculo. Quito: EMS.

Hernández, R., Fernández, C. y Baptista, P. (2010). Metodología de la investigación. México, D.F.: Editorial Mc Graw Hill.

Instituto Colombiano para el Fomento de la Educación Superior - ICFES, (2016). Resultados pruebas saber 3, 5 y 9 del Municipio de Fundación Departamento del Magdalena. Bogotá, D.C.: ICFES.

Jiménez, J. y Salas, M. (2017). Aplicación de modelos econométricos para estimar la aceptabilidad de una tasa por congestión vehicular. INGE CUC, 13(2), 60-78. https://doi.org/10.17981/ingecuc.13.2.2017.08

Nuñez, P. (2004). Educación lúdica: técnicas y juegos pedagógicos. Bogotá, D.C.: San Pablo.

República de Colombia. Ministerio de Educación Nacional - MEN. (2016). Derechos Básicos de Aprendizajes (DBA). Segunda Versión. Bogotá, D.C.: MEN. 
República de Colombia. Ministerio de Educación Nacional - MEN. (2016b). Estándares Básicos de Competencias en Lenguaje, Matemáticas, Ciencias y Ciudadanas. Bogotá, D.C.: MEN. Recuperado de https://www.mineducacion. gov.co/1621/articles-340021_recurso_1. pdf
Reveco, O. (2005). Desarrollo infantil y Metodologías para el Aprendizaje. Quito: Editorial AFEFCE.

Vygotsky, L. (1977). Pensamiento y lenguaje. Teoría del desarrollo cultural de las funciones psíquicas. Buenos Aires: La Pléyade.

Vygotsky, L. (1979). Desarrollo de los procesos psicológicos superiores. Barcelona: Grijalbo-Crítica. 